\title{
BOUVERET'S SYNDROME (CASE PRESENTATION)
}

Dipak Ladda ${ }^{1}$

\section{HOW TO CITE THIS ARTICLE:}

Dipak Ladda. "Bouveret's syndrome (case presentation)". Journal of Evolution of Medical and Dental Sciences 2014; Vol. 3, Issue 04, January 27; Page: 855-862, DOI:10.14260/jemds/2014/1915

ABSTRACT: Bouveret's syndrome is defined as gastric outlet obstruction caused by duodenal impaction of a large gallstone which passes into the duodenal bulb through a cholecystogastric or cholecystoduodenal fistula. We describe a case of Bouveret's Syndrome in an middle aged patient that was successfully treated with resection of distal part of stomach and $1^{\text {st }}$ part of duodenum by laparotomy. We will also review the literature on this uncommon condition. Biliary enteric fistula is as such a rare complication and comparatively rare cause of intestinal obstruction. And even if becomes so; generally it lodges in the terminal ileum and rarely in duodenum. Obstructing proximal part of duodenum or distal part of stomach is a curiosity and i.e. Bouveret's syndrome. Generally patient presents with nausea, vomiting, haematemesis, abdominal distention or epigastric pain. G.I. bleeding, obstructive jaundice, pancreatitis or duodenal or gastric perforation are rare complications. The treatment is purely surgical - either enterolithotomy or gastrotomy with associated cholecystectomy with repair of fistula or enterolithotomy with or without second stage cholecystectomy. It is worth to mention here that so far histological findings of Bouveret's syndrome are not described in the literature. Even though findings are not that much specific; but presence of foci of abscesses, dense mixed inflammatory exudates in all the coats of distal part of stomach, $1^{\text {st }}$ part of duodenum and gall bladder along with formation of lymphoid follicles with prominent germinal centers in muscle coat are definitely mentionable and we feel these may give clue. On gross examination the thickness of stomach wall and duodenal wall are increased and there is narrowing of lumen. If there is stone in gall bladder; it may also show thickened out wall due to presence of chronic cholelithiasis with cholecystitis.

1. INTRODUCTION: Leon Bouveret reported this condition first time in 1896 on two patients. [1].It is definitely rare entity; but now reports are available in literature; and that too are specifically described and ascribed to elderly population and they are common in females (65\%) with a median age of 74.1 years at presentation [2].Because it often presents in patients with advanced age and multiple comorbidities, it is associated with a high rate of mortality. And because of this reason it is always advocated to attempt endoscopic treatment to make bypass to surgery. For patients in whom endoscopic extraction has failed, simple enterolithotomy, duodenotomy or gastrotomy, and stone extraction can be performed [3].

\section{CASE PRESENTATION:}

2.1 History: Patient presented with history of off and on abdominal pain started $1 \mathrm{yr}$ back. He never had severe pain anytime. No vomiting or jaundice anytime. Patient consulted at one institute $1 \mathrm{yr}$ back. CT scan done at that time showed mass involving the fundus of the gall bladder infiltrating the pylorus and hepatic flexure. Multiple nodes in hepatoduodenal ligament and along the celiac axis. FNAC done at that time showed no malignant cells; however in view of CT findings he was diagnosed 
as advanced Ca gall bladder for which no therapy was given. Patient continued to have off and on abdominal pain.

2.2 Examination: Now this time he presented with gastric outlet obstruction. Upper GI endoscopy showed narrowing in first part of duodenum without any growth. CT scan done again; which showed mass involving the gall bladder, duodenum and colon. However no definite nodes or liver lesion found. Surgery planned in view of gastric outlet obstruction.
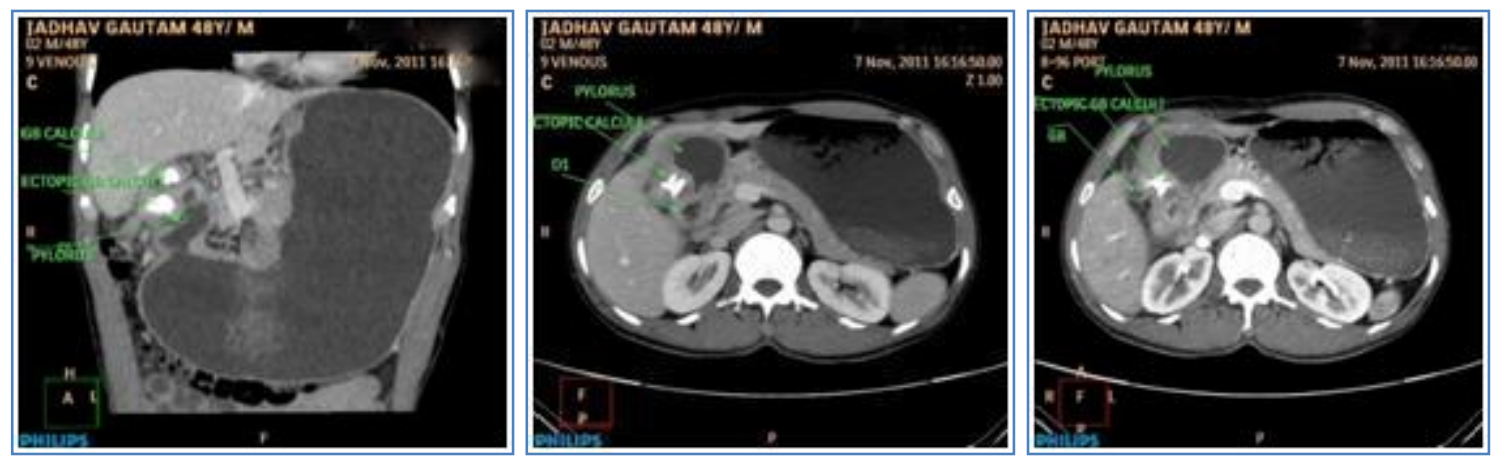

2.3 Intraoperative Findings \& Surgery Performed: Intra-operative findings.... mass involving the gall bladder fundus with fistula in 1st part duodenum and hepatic flexure of colon. Node along hepatic artery was present. Cholecystectomy along with resection of distal part of stomach and 1st part of duodenum done. Opening in colon at fistula site refashioned and closed in 2 layers. Celiac node taken for biopsy.

Operative findings were more in favour of benign disease in view of lot of inflammatory tissue all around the duodenum and gall bladder; and survival of the patient for $1 \mathrm{yr}$ after suspecting the advanced malignancy initially. Because average survival of Ca gallbladder after diagnosis where surgery not possible is 6 to 8 months.

On physical exam, patient was in no acute distress, afebrile, and hemodynamically stable. Pertinent findings included mild tenderness to palpation in the epigastric area, an audible succession splash and normal bowel sounds. His liver enzymes, electrolytes, and creatinine level were all within normal limits.

Gross Examination: The specimens received are distal part of stomach, $1^{\text {st }}$ part of duodenum, gall bladder and celiac lymph node. On cutting open the gall bladder; blackish calculus is seen to be impacted in the lumen quiet adherent and invading the wall. It measures $1.5 \times 1.3 \mathrm{cms}$. Cut section through duodenum shows thickened out wall and narrowed lumen. Cut section through distal part of stomach shows impacted stone in the outer wall of stomach within a blind fistulous tract. The wall of stomach is markedly thickened and literally obliterates the lumen. 


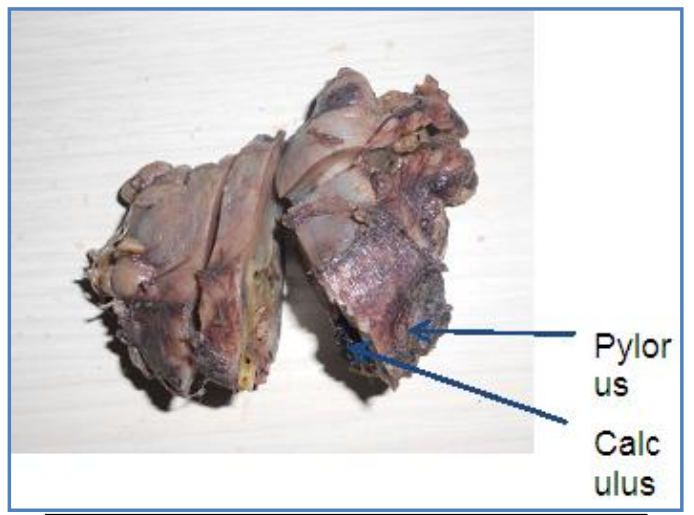

Cut opened distal part of stomach

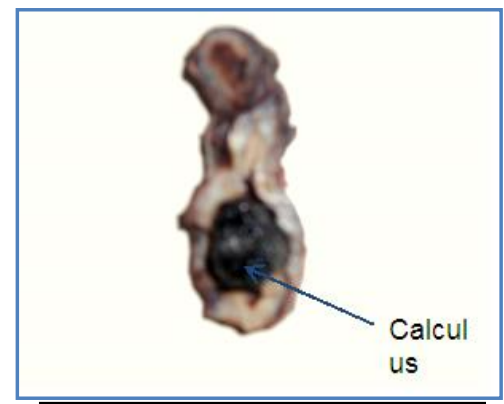

Cut opened gall bladder

Microscopic Examination: Sections from stomach and duodenum show thickened out muscle coat with dense lymphocytic infiltrate with formation of lymphoid follicles along with prominent germinal centers. Besides these; there is diffuse and dense mixed inflammatory exudates and foci of abscesses. Sections from gall bladder also show similar findings as that of stomach.

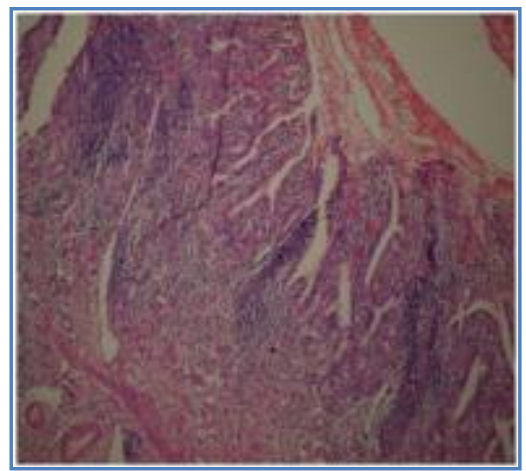

Section from pylorus

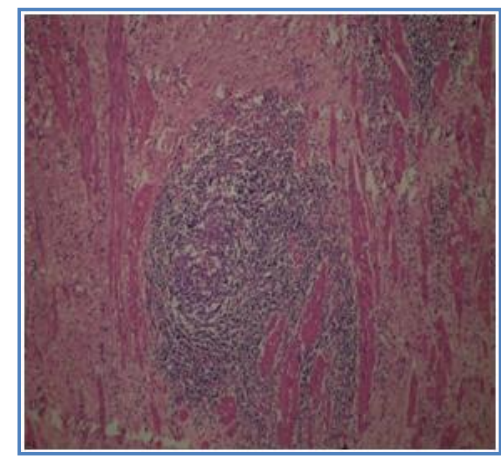

Lymphoid follicles in muscle coat

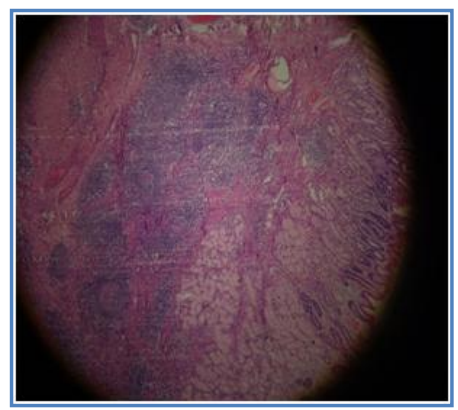

Section of duodenum - Lymphoid follicles in muscle coat

3. DISCUSSION: Cholelithiasis is not an uncommon problem; by age 75 , about $35 \%$ of women and $20 \%$ of men have developed gallstones [4]. Out of these only a small percentage of patients (approximately 6\%) develop complications including such rare complications as 


\section{CASE REPORT}

cholecystoduodenal fistulas [5]. It results due to chronic inflammation, reduced arterial blood supply and stagnation of venous blood with resultant adhesions between the gallbladder and the bowel wall. [6]. And it is due to pressure necrosis and compression of a gallstone against the gallbladder wall, end result ends with fistula formation and subsequent passage of gallstones via the fistula can then result in gallstone ileus [7]. The frequency in which gall stone can stuck after passing through the fistula in descending order are ileum (90\%), colon (3-8\%) and duodenum (3\%). It is this rare condition which is called as Bouveret's Syndrome. (8). The syndrome was first described by Beaussier in 1770 and was subsequently named after the French physician Leon Bouveret after he published two case reports in Revue de Medecin in 1896.The differential diagnosis of gastric outlet obstruction includes diverticulae, foreign bodies, fibrotic ulcers and neoplasia]. More common in elderly women, Bouveret's syndrome presents with a non specific triad of epigastric pain, nausea and vomiting. Abdominal and chest radiographs should be performed looking for evidence of aerobilia, bowel obstruction and ectopic gallstones. Abdominal CT should also be performed. Typical findings on OGD include a dilated stomach and a hard non-fleshy mass at the obstruction. Abdominal ultrasound or computerized tomography was diagnostic in about $60 \%$ of cases. (9). In most cases Bouveret's syndrome is treated surgically. The options available are either a single-staged enterolithotomy (or gastrotomy) with concomitant cholecystectomy and repair of the fistula; or (b) an enterolithotomy alone with or without a second-stage cholecystectomy. Endoscopic extraction of the stone is described in selected patients in whom it is technically possible to dislodge and engage the stone in a Dormia basket. (10, 11). Extracorporeal shockwave lithotripsy7, electrohydrauliclithotripsy8, and laser lithotripsy9 have also been successfully used to fragment large stones.

3.1 Diagnosis: Generally patients present without specific signs and symptoms. However; Cappell and Davis described the most common symptoms of patients with Bouveret's syndrome as nausea and vomiting (86\%), and abdominal pain (71\%); less commonly, patients present with hematemesis, weight loss, and anorexia [2]. General examination may often show abdominal tenderness, abdominal distention and dehydration. Now a days the diagnosis may be offered even with gastroscopy (12). Endoscopy, may show a stone causing obstruction in about $69 \%$ of patients and obstruction in the absence of a visualized stone or fistula is seen in $31 \%$ of cases [2]. In the remaining cases, the stone may not actually be visualized because it is compressing the lumen and only partially visualized through the duodenal wall. Other findings described include excessive retained food or fluid in the stomach and inflammation, edema, or ulcer at the impacted site [2]. CT is diagnostic in about $60 \%$ of cases and is helpful in demonstrating the exact level of obstruction, the biliary site of the duodenal fistula, and the status of the gallbladder [13, 14]. Approximately 15-25\% of gallstones are isoattenuating and not well visualized on CT. In such cases, magnetic resonance cholangiopancreatography (MRCP) may be useful, because it may more clearly delineate fluid from calculi.

3.2 Treatment: Endoscopic extraction, endoscopic laser lithotripsy (ILL), extracorporeal shockwave lithotripsy (ESWL), and intracorporeal electrohydraulic lithotripsy (IEHL) have all been reported as alternatives to surgery for more proximal gallstone obstruction whereas surgery is routinely recommended for individuals with impaction of the gallstone more distally (gallstone ileus). 
Endoscopic treatment of Bouveret's syndrome should be considered a first-line option despite the low success rate reported in the literature. The first successful endoscopic extraction was described in 1985 by Bedogni et al. [15]. Subsequently, a number of case reports have been published describing successful endoscopic management of Bouveret's syndrome $[3,6,16]$. Endoscopic management often necessitates the use of different sized and shaped snares, grasping forceps, retrieval baskets and nets, biliary balloons, and sometimes even a side-viewing endoscope; it can be technically challenging, time-consuming, and success rates in case series have been previously reportedly to be less than $10 \%$ [3].

Endoscopic treatment, accompanied by lithotripsy using a variety of different modalities, has been well described. There are several reports of intracorporeal laser lithotripsy (ILL) alone, and in combination with, ESWL $[6,15,16]$. The first reported successful use of ILL was in 1999 by Maiss et al. requiring a total of nine sessions [16]. The drawbacks of this particular procedure are the need for prolonged and multiple sessions, and the risk of converting a proximal gallstone ileus into a distal gallstone ileus (as a result of partial fragmentation of the stone).

IEHL can be used alone or in combination with other methods. Moriai et al. used IEHL with mechanical lithotripsy for removal of two $3 \mathrm{~cm}$ stones in 1991 [18]. In 1997 Dumonceau et al. reported a case of successful treatment with IEHL after failure of ESWL [19]. Two additional cases described by Huebner et al., in 2007 saw the incorporation of IEHL as the sole modality with successful stone extraction [20]. The risk involved with this method is that inadvertent focusing of the shockwaves onto the intestine wall may cause bleeding and perforation.

Extracorporeal shockwave lithotripsy (ESWL) has also been used with success in treating patients with Bouveret's syndrome [19,21-23]. Limitations of using ESWL include the need for several return sessions, in addition to eventual endoscopy. Also, ESWL may be difficult to perform in obese patients or if there are gas-containing bowel loops interposed between the gallstone and the abdominal [19, 21-23].

In general, the success rate of endoscopic extraction is dependent on stone size. Stones, that are larger than $2.5 \mathrm{~cm}$ are more difficult to extract endoscopically, although extractions of stones up to $3 \mathrm{~cm}$, have been reported [3]. Larger stones can cause ischemic ulceration of the adjacent duodenal wall. Moreover, these stones tend to have a hard outer shell and soft inner core making mechanical fragmentation with endoscopic forceps or laser more difficult. While the majority of patients tolerate attempted endoscopic treatment, there has been a case report of pulseless electrical activity (PEA) during mechanical retrieval due to the gallstone getting lodged in the esophagus; the PEA abruptly resolved when the stone was pushed back into the stomach. In a handful of cases, surgery was needed subsequent to upper endoscopy due to stricture, sepsis, and a second stone in the duodenum [24].

If endoscopic treatment fails, the patient will require surgical management. Forty-two percent of surgical patients have previously undergone a failed endoscopic treatment. Surgical options include a combination of enterolithotomy (removal of the stone) plus cholecystectomy and fistula repair, but surgery is associated with significant morbidity and mortality [25-29]. Combined treatment has been associated with a higher mortality of $20-30 \%$, compared to just $12 \%$ in cases of simple duodenotomy. Laparoscopy is also an additional option for surgical treatment; Sica et al. reported, in 2005, the first case of uneventful stone removal and cholecystectomy by laparoscopy [29]. 
Although debatable, fistula repair in patients treated with endoscopic methods or simple enterolithotomy is often considered unnecessary due to spontaneous closure, especially when the cystic duct is patent and no residual stones are present. On the other hand, the persistence of symptoms, the possibility for recurrence, and the risk for gallbladder cancer lend support to fistula repair.

4. CONCLUSION: So far histological findings of Bouveret's syndrome are not described in literature. It may be possible that it may be because of variation in the impaction of stone either in lumen or in different portions of wall of stomach or duodenum or somewhere else like in fistulous tract itself. However; it is because of long duration of impaction of stone; the surrounding tissue reacts to it and there is inflammatory and immunological response. Due to this there is mixed inflammatory response, foci of abscesses formations and lymphoid follicle along with prominent germinal center formation in muscle coat. Even though the histological findings may not be seen to be specific with a single case study; I feel if we keep attention with similar type of findings further in forthcoming cases; those may guide us to reach to some conclusive findings in future.

\section{REFERENCES:}

1. L. Bouveret, "Stenose du pylore adherent a la vesicule," Revue Medicale (Paris), vol. 16, pp. 116, 1896.

2. M. S. Cappell and M. Davis, "Characterization of Bouveret's syndrome: a comprehensive review of 128 cases," The American Journal of Gastroenterology, vol. 101, no. 9, pp. 2139-2146, 2006.

3. A.S. Lowe, S. Stephenson, C. L. Kay, and J. May, "Duodenal obstruction by gallstone (Bouveret's syndrome): a review of the literature," Endoscopy, vol. 37, no. 1, pp. 82-87, 2005.

4. H. F. Newman and J. D. Northup, "The autopsy incidence of gallstones," Surgery, Gynecology \& Obstetrics, vol. 109, no. 1, pp. 1-13, 1959.

5. K. A. LeBlanc, L. H. Barr, and B. M. Rush, "Spontaneous biliary enteric fistulas," Southern Medical Journal, vol. 76, no. 10, pp. 1249-1252, 1983.

6. J. Langhorst, B. Schumacher, T. Deselaers, and H. Neuhaus, "Successful endoscopic therapy of a gastric outlet obstruction due to a gallstone with intracorporeal laser lithotripsy: a case of Bouveret's syndrome," Gastrointestinal Endoscopy, vol. 51, no. 2, pp. 209-213, 2000.

7. P. J. Pickhardt, J. A. Friedland, D. S. Hruza, and A. J. Fisher, "CT, MR cholangiopancreatography, and endoscopy findings in Bouveret's syndrome," American Journal of Roentgenology, vol. 180, no. 4, pp. 1033-1035, 2003.

8. F. M. Frattaroli, D. Reggio, A. Guadalaxara, G. Illomei, D. Lomanto, and G. Pappalardo, "Bouveret's syndrome: case report and review of the literature," Hepato-Gastroenterology, vol. 44, no. 16, pp. 1019-1022, 1997.

9. Cappell MS, Davis M. Characterization of Bouveret's syndrome: A comprehensive review of 128 cases. Am J Gastroenterol 2006;101:2139-2146.

10. Bedogni G, Contini S, Meinero M, Pedrazzoli C, Piccini GC. Pyloroduodenal obstruction due to a biliary stone (Bouveret's syndrome) managed by endoscopic extraction. Gastrointest Endosc 1985;31:36-38

11. Torgerson SA, Greening GK, Juniper K, Farrell RL. Gallstone obstruction of the duodenal cap (Bouveret's syndrome) diagnosed by endoscopy. Am J Gastroenterol 1979;72:165-167. 
12. 0. Grove, "Acute pyloric obstruction by gallstone: report of a case diagnosed by gastroscopy,"Gastrointestinal Endoscopy, vol. 22, no. 4, pp. 212-213, 1976.

13. D. Tüney and C. Çimşit, "Bouveret's syndrome: CT findings,” European Radiology, vol. 10, no. 11, pp. 1711-1712, 2000.

14. S. Trubek, J. K. Bhama, and N. Lamki, "Radiological findings in bouveret's syndrome," Emergency Radiology, vol. 8, no. 6, pp. 335-337, 2001.

15. G. Bedogni, S. Contini, M. Meinero, C. Pedrazzoli, and G. C. Piccinini, "Pyloroduodenal obstruction due to a biliary stone (Bouveret's syndrome) managed by endoscopic extraction," Gastrointestinal Endoscopy, vol. 31, no. 1, pp. 36-38, 1985.

16. J. Maiss, J. Hochberger, S. Muehldorfer, J. Keymling, E. G. Hahn, and H. T. Schneider, "Successful treatment of Bouveret's syndrome by endoscopic laserlithotripsy," Endoscopy, vol. 31, no. 2, pp. S4-S5, 1999.

17. M. M. Alsolaiman, C. Reitz, A. T. Nawras, J. B. Rodgers, and B. J. Maliakkal, "Bouveret's syndrome complicated by distal gallstone ileus after laser lithotropsy using Holmium: YAG laser," BMC Gastroenterology, vol. 2, article 15, pp. 1-4, 2002.

18. T. Moriai, T. Hasegawa, M. Fuzita, A. Kimura, T. Tani, and I. Makino, "Successful removal of massive intragastric gallstones by endoscopic electrohydraulic lithotripsy and mechanical lithotripsy," American Journal of Gastroenterology, vol. 86, no. 5, pp. 627-629, 1991.

19. J.-M. Dumonceau, M. Delhaye, J. Devière, M. Baize, and M. Cremer, "Endoscopic treatment of gastric outlet obstruction caused by a gallstone (Bouveret's syndrome) after extracorporeal shock-wave lithotripsy," Endoscopy, vol. 29, no. 4, pp. 319-321, 1997.

20. E. S. Huebner, S. DuBois, S. D. Lee, and M. D. Saunders, "Successful endoscopic treatment of Bouveret's syndrome with intracorporeal electrohydraulic lithotripsy," Gastrointestinal Endoscopy, vol. 66, no. 1, pp. 183-184, 2007.

21. C. Gemmel, U. Weickert, A. Eickhoff, D. Schilling, and J. F. Riemann, "Successful treatment of gallstone ileus (Bouveret's syndrome) by using extracorporal shock wave lithotripsy and argon plasma coagulation," Gastrointestinal Endoscopy, vol. 65, no. 1, pp. 173-175, 2007.

22. P. Ondrejka, "Bouveret's Syndrome treated by a combination of extracorporeal shock-wave lithotripsy (ESWL) and surgical intervention," Endoscopy, vol. 31, no. 9, p. 834, 1999.

23. J. Holl, M. Sackmann, R. Hoffmann, et al., "Shock-wave therapy of gastric outlet syndrome caused by a gallstone," Gastroenterology, vol. 97, no. 2, pp. 472-474, 1989.

24. J. Moschos, I. Pilpilidis, Z. Antonopoulos, et al., "Complicated endoscopic management of Bouveret's syndrome. A case report and review," Romanian Journal of Gastroenterology, vol. 14, no. 1, pp. 75-77, 2005.

25. J. C. Rodríguez-Sanjuán, F. Casado, M. J. Fernández, D. J. Morales, and A. Naranjo, "Cholecystectomy and fistula closure versus enterolithotomy alone in gallstone ileus," British Journal of Surgery, vol. 84, no. 5, pp. 634-637, 1997.

26. J. K. Bhama, J. W. Ogren, T. Lee, and W. E. Fisher, “Bouveret's syndrome," Surgery, vol. 132, no. 1, pp. 104-105, 2002.

27. R. M. Reisner and J. R. Cohen, “Gallstone ileus: a review of 1001 reported cases," American Surgeon, vol. 60, no. 6, pp. 441-446, 1994.

28. N. Zuegel, A. Hehl, F. Lindemann, and J. Witte, "Advantages of one-stage repair in case of gallstone ileus,” Hepato-Gastroenterology, vol. 44, no. 13, pp. 59-62, 1997. 


\section{CASE REPORT}

29. G. S. Sica, P. Sileri, and A. L. Gaspari, "Laparoscopic treatment of Bouveret's syndrome presenting as acute pancreatitis," Journal of the Society of Laparoendoscopic Surgeons, vol. 9, no. 4, pp. 472-475, 2005.

\section{AUTHORS:}

1. Dipak Ladda

\section{NAME ADDRESS EMAIL ID OF THE CORRESPONDING AUTHOR:}

Dr. Dipak Ladda,

$1^{\text {st }}$ Floor, Vidate Complex,

Solapur Highway, Opp. Hotel Krishnachaya,

Hadapsar, Pune - 411028.

\section{PARTICULARS OF CONTRIBUTORS:}

1. Histopathologist, Department of Pathology, Chennai Medical College, Hospital \& Research Centre, Trichi, Tamilnadu.

E-mail: drladdadipak@yahoo.com

Date of Submission: 17/10/2013.

Date of Peer Review: 18/10/2013.

Date of Acceptance: 29/10/2013.

Date of Publishing: 21/01/2014 